\title{
Estandarización de los procedimientos para la dosificación de líquidos para la formulación de alimentos balanceados para aves
}

\author{
Standardization of procedures for liquid dosing for the formulation of \\ feed for poultry
}

\author{
Maikel José Velásquez Marcano \\ maikel.velasquez08@gmail.com
}

Universidad Nacional Experimental de las Fuerzas Amadas, Venezuela

\section{RESUMEN}

Con el propósito de garantizar la calidad de los productos elaborados por la empresa Alimentos La Caridad C.A, en la producción de alimentos balanceados para aves, se propuso la estandarización de los procedimientos para dosar líquidos en sus mezcladoras. La metodología consistió, en líneas generales, en la identificación de las características de las materias primas y productos terminados según su uso, realizar muestreo y pruebas necesarias a las materias primas. Los resultados se muestran en cuatro fases; diagnóstico, evaluar los procesos, proponer procedimientos y verificar la factibilidad. Entre las conclusiones se encuentran que el proceso de dosificación consiste en estandarizar la desviación que existe al agregar los compuestos líquidos y que el error sea menor, los líquidos utilizados son la colina, metionina y las grasas cada uno de ellos cumple un papel importante para la absorción y aprovechamiento de los nutrientes promovido por los cereales, las vitaminas o pre-mezclas y los polvos agregados.

Palabras clave: Alimentos balanceados para aves; Aseguramiento de la calidad; estandarizar; gallinas; gallus gallus domesticus

\section{ABSTRACT}

In order to guarantee the quality of the products made by the company Alimentos La Caridad C.A, in the production of feed for poultry, the standardization of the procedures for dosing liquids in their mixers was proposed. The methodology consisted, in general, in the identification of the characteristics of the raw materials and finished products according to their use, to carry out sampling and necessary tests to the raw materials. The results are shown in four phases; diagnosis, evaluates the processes, propose procedures and verify the feasibility. Among the conclusions are that the dosing process consists in standardizing the deviation that exists when adding the liquid compounds and that the error is smaller, the liquids used are choline, methionine and fats each of them plays an important role for the absorption and utilization of nutrients promoted by cereals, vitamins or pre-mixtures and added powders.

Key words: Balanced food for poultry; quality assurance; standardization; chickens; gallus gallus domesticus 
INTRODUCCIÓN

Existe una gran variedad de alimentos que pueden ser utilizados para las aves (Castello, 1977). Su selección es función de las necesidades fisiológicas y procesos de crecimiento. Estos alimentos deben ser fuente de energía y ricos en multivitamínicos, para así obtener mejor rendimiento y mayor reproducción (Valdivié et al., 2008). Los alimentos concentrados deben ser altos en contenido de fósforo, calcio, sodio, vitaminas, minerales, entre otros, estos elementos coadyuvarán al ave y a sus crías o huevos, en el caso de las ponedoras (Farfán, 2013).

A menudo se utilizan diferentes raciones, dependiendo de la fase de producción del ave. Las raciones de inicio son altas en proteína, un ingrediente costoso en la alimentación (Acosta, 2006). Sin embargo, las raciones de crecimiento y acabado pueden ser bajas en proteínas, ya que las aves mayores requieren menos cantidad. Una dieta adecuada tiene que tener 24,20 y $18 \%$ de proteínas al inicio, durante el crecimiento $20 \%$ del ave y de acabado, respectivamente (Hidalgo, 2015). Los alimentos para gallinas ponedoras generalmente tienen alrededor de $16 \%$ de proteína. Hay raciones especiales disponibles para pollos de engorde, pollitas, ponedoras y reproductores (Fonseca y Fonseca, 2011).

Según Martín (2005), el diseño de programas de alimentación para una especie y/o producción determinada, tendrá como base las características genéticas, los objetivos productivos, aspectos comerciales y los rendimientos económicos buscados. En definitiva estará ligada a las características y demanda del mercado.
La empresa Alimentos La Caridad C.A. es una institución dedicada a la fabricación de productos alimenticios para aves de tipo Gallus Domesticus (Vallortigara et al., 1990) y de género gallinas reproductoras o ponedoras, donde sus ingredientes activos principales son el maíz en grano y el frijol o harina de soya (Marín Gómez y Benavides, 2007; Castillo, 2017; Mendoza, 2016). Los alimentos formulados dentro de esta industria son preposteras, ponedoras especiales, ponedoras normales, ponedoras fase $2 \mathrm{y}$ pollita $\mathrm{y}$ pollona.

El proceso de transformar la materia prima a los productos terminados se realiza por etapas, existiendo diferencias cuando son distintos productos, ya sea por la variación de los parámetros físicos y químicos y de acuerdo a los ingredientes empleados, rigiéndose por las fórmulas realizadas por los nutricionistas encargados de estudiar los nutrientes, vitaminas y minerales de cada uno de los alimentos arriba mencionados. En general, el procedimiento para elaborar los alimentos reproductores se inicia en la etapa de recepción, donde se almacena, controla y despacha las materias primas y las necesarias para el proceso productivo, dirigido a las diversas áreas de planta bajo planificación y requerimiento.

Las materias primas resguardadas en el Almacén de Materias Primas son trasladadas a la primera etapa de transformación, donde se realiza un proceso de agregación dentro de las tolvas que van dirigidas a las mezcladoras. En éstas se agregan materias primas variadas, teniendo de guía las fórmulas recetadas por el nutricionista y de acuerdo con la especificación de los productos terminados. 
En los ingredientes se encuentran los líquidos, que son parte fundamental de una alimentación balanceada de las aves. Este tiene un proceso gradual interno dentro de las homogeneizadoras o mezcladoras del alimento que se debe controlar y estudiar (Meleán-Romero et al., 2008). En este sentido, la empresa cuenta con un departamento encargado de verificar el buen funcionamiento en la adición de las dosis líquidas requeridas. Este es el Departamento de Aseguramiento de Calidad e, implícitamente, se encargan de verificar la agregación necesaria según lo estipulado para cada tipo de alimento.

El Departamento de Aseguramiento de Calidad cuenta con personal técnico con conocimiento y experiencia en las dosificaciones de líquidos, que se deben incorporar en las mezcladoras. Dentro de estos fluidos se encuentra la colina, grasas y metionina o alimeth, utilizadas para la fabricación de alimentos para aves con nutrientes vitamínicos balanceados. Para realizar este procedimiento se utilizan métodos y técnicas que hacen que esta actividad obtenga un alto nivel de confiabilidad en las muestras obtenidas.

La dosificación o chequeo de los líquidos son procesos que tienen como función establecer un parámetro de control, consintiendo asegurar la calidad. Este permite verificar que las cantidades agregadas son las necesarias para producir el alimento, teniendo una desviación permitida del $\pm 2 \%$. El proceso de dosificación de los líquidos que van a las mezcladoras no poseen un patrón a seguir. Es decir, cada operador tiende, de manera natural y casi inconsistente, a desarrollar técnicas desde su punto de vista o criterios propios, lo que provoca indiscutiblemente que el procedimiento o métodos para evaluar los niveles de dosificación, no sean los esperados y las técnicas no sean las indicadas o se hagan más tediosas. En este sentido, el Departamento de Aseguramiento de Control de Calidad se ha planteado realizar la estandarización de procesos y técnicas para el chequeo de los líquidos donde se especifique los pasos a realizar, este método no se encuentra establecido a nivel de laboratorio.

El propósito de este trabajo es diseñar el proceso de estandarización de los procedimientos para la dosificación de los líquidos para la formulación de alimentos balanceados para aves.

\section{MATERIALES Y MÉTODOS}

\section{a) Identificación de las carac- terísticas de las materias primas y productos terminados según su uso}

Se verifica en el manual de análisis físicos a realizar y se plasman los procedimientos de cada materia prima. Las materias primas deben poseer un estándar de calidad para asegurar la del producto final. Así mismo se identifican características de los productos terminados destinados a las granjas clientes, según el uso que vaya a ser destinado su consumo en la etapa de desarrollo de las ponedoras, esta característica de consumo viene dada por su edad. Los alimentos son producidos según la formulación nutricional para cada etapa de desarrollos de las aves, es decir, la cantidad a agregar de cada materia prima, el grosor del maíz y la cantidad de soya a utilizar, entre otros. 


\section{b) Realizar muestreo y pruebas necesarias a las materias primas}

Las materias primas con estándares de calidad son las necesarias para la formulación de alimentos balanceados. Para esto, se tienen que realizar pruebas a las materias primas que ingresan a la empresa distribuida por los distintos proveedores. Los más empleados en estado sólido son: granos, harinas y polvos, y los líquidos. Las materias primas utilizadas en su mayoría son de origen nacional y productos de importación de países como Argentina, Estados Unidos (USA), Brasil, entre otros. En este sentido, dentro de las materias primas sólidas están los granos grandes como son: maíz amarillo o blanco y frijol de soya crudo o desactivado, a los cuales se les realizan pruebas de humedad, densidad $\mathrm{y}$ tipificación donde se refleja en porcentajes de granos partidos, dañados, amiláceos, enteros y el porcentaje de impurezas, Esta prueba se realiza en una muestra tomada al camión con una cantidad parcial de 500 g de la total. Así mismo, se tienen las harinas, granos finos a los cuales están inmersos los carbonatos de calcio gruesos y finos, el cloruro de sodio o sal común, harinas de carne y soya de importación, a estos se le realizan evaluaciones de densidad sin golpe y compactada y granulometría en el tamiz Ro-Tap (Fuentes y Heber, 2016).

Para dar continuidad a las materias primas utilizadas en la dieta balanceada de un ave se tienen las vitaminas ponedoras, pollitas y reproductoras, minerales ponedoras, larvakill, ambiflux, fosfato bicalcio, zeolitech entre algunos otros. En su mayoría los polvos son producidos en el departamento de pre-mezcla y no se les realizan pruebas, ya que posee un laboratorio independiente. Finalmente, se tienen los líquidos que son grasa como oleína, aceite crudo de soya, aceite de palma y grasa amarilla y sólo se muestrean $y$ son enviadas al laboratorio de aseguramiento de la calidad químico.

\section{c) Efectuar la toma de muestras de productos terminados a cada camión con destino a las granjas}

A todos los camiones con destino a las granjas se les toma muestra del alimento que va a ser transportado por petición de los clientes. Su zona de carga tiene 14 silos con contenidos de alimentos concentrados. La muestra se toma en bolsas plásticas de $2,5 \mathrm{~kg}$, identificada con el nombre del chofer y el número del silo. Es conveniente resaltar que, según las normas COVENIN 1567-80 (1980), se establece que los métodos de muestreos en camiones transportadores de alimentos para aves se realizan con un calador de tipo cilíndrico con aberturas, de longitud apropiada, y diámetro de 4 a $5 \mathrm{~cm}$. Las tomas de muestras varían según el tamaño del camión y la capacidad del remolque, según se muestra en la Tabla 1. De igual manera, la norma establece este muestreo debido a que las mezcladoras son de 2.000 y 2.500 kg y se realizan varias hasta llenar el silo 
Tabla 1. Número de muestras en función de la capacidad del camión

\begin{tabular}{c|c}
\hline $\begin{array}{c}\text { Capacidad del camión } \\
\text { (ton) }\end{array}$ & $\begin{array}{c}\text { No. de puntos } \\
\text { distintos }\end{array}$ \\
\hline $0-15$ & Cinco (5) \\
$15-30$ & Ocho (8) \\
$30-50$ & Once (11) \\
\hline
\end{tabular}

Con las muestras en el laboratorio se procede a tomar registro de los datos del viaje, alimento transportado, fecha del traslado, mezcladora que realiza el alimento, silo del cual proviene, granja destino y chofer encargado del transporte. Además, pasa a una segunda etapa donde se mezcla el alimento y se realizan las pruebas de densidad y densidad compactada para luego pesar $200 \mathrm{~g}$ de la muestra y llevarlo al tamiz Ro-Tap para obtener su granulometría. Luego se procede a preparar una muestra que va para análisis químicos moliendo una pequeña cantidad ya haciéndolo pasar por la malla $\mathrm{N}^{\circ} 18$ del tamizador y etiquetarla con una codificación especifica que se maneja en el área de trabajo. Finalmente, se envasa una muestra testigo, que funciona a manera de respaldo en caso de un inconveniente con el cliente.

\section{d) Verificar los molinos 1 y 2}

Dentro de la empresa existen dos mezcladoras y dos molinos. El molino 1 es la alimentación del maíz para la mezcladora $1 \mathrm{y}$, de igual manera, el molino 2 alimenta la mezcladora 2. Los molinos funcionan con unas cribas que parten el maíz a través de la fuerza de impulsión y con los golpes el maíz es partido. Esta prueba se realiza tres veces al día, como método de verificación del proceso. Esto consiste en tomar una muestra de cada uno de los molinos abriendo una compuerta de descarga, la misma es llevada al laboratorio con el fin de realizar análisis y pasarlo por el tamiz Ro-Tap para obtener su granulometría y se calcula la densidad de este maíz y registran los correspondiente a estos valores. Este proceso es denominado molienda (Tovar y Colonia, 2013). La molienda se estudia tres veces al día para determinar si existen granos enteros o si hay gran cantidad de granos gruesos y enteros. Esto es señal de avería en el molino y debe ser notificados en el departamento de mecánica. Posterior a la inspección, se repiten las pruebas con una nueva muestra para verificar la solución de la avería. Si el alimento posee granos de grosor significativo (Antelo, 2008), las aves ponedoras no lo consumen, ya que son animales selectivos, y bajan la postura de huevos, generando baja en la entrada de dinero a la granja.

\section{e) Ejecutar las pruebas de hume- dades a los granos}

La humedad no es más que la cantidad de agua presente en un material con respecto a su masa total o respecto a la seca. En los granos, la cantidad de agua presente afecta sus propiedades por lo importante chequear su humedad $\mathrm{y}$ controlar su cantidad de agua, ya que son un material higroscópico que realizan intercambio con el ambiente. Si la humedad no es controlada, el método de almacenamiento no es seguro, es decir, si los granos poseen alto grado de humedad tienden a sufrir descomposición dañando 
su fibra interna, obtener hongos o generar infestaciones dentro del tanque de almacenamiento (silo) (Martínez et al., 2010).

Para determinar la humedad, en laboratorio se toma una muestra de $250 \mathrm{~g}$ del grano (maíz o frijol de soya) y se codifica. A través de la bandeja de entrada se introduce la muestra y se espera aproximadamente de 4 a $6 \mathrm{~s}$ y el valor es arrojado en la pantalla en porcentaje de humedad, este equipo es calibrado por especialistas cada seis meses. Adicionalmente, se realiza la prueba o test rojo fenol a la soya. Esta posee una enzima llamada ureasa que a través de su descomposición forma amoniaco y $\mathrm{CO}_{2}$ los mismos son tóxicos para el organismo. La empresa importa el frijol de soya, la cual contiene alto contenido en esta enzima, ya que el mismo se encuentra en su estado natural dentro de la planta. Otra planta llamada desactivadora de soya, que realiza un proceso de cocción al frijol a través de un arrastre de vapor de agua producido por una caldera, desactiva las enzimas productoras de amoniaco y $\mathrm{CO}_{2}$. $\mathrm{La}$ disolución de amoniaco tiene un $\mathrm{pH}$ alto, por ello se puede ser detectado mediante una pruebo con una solución de rojo fenol [18]. La manera de realizar este análisis es, colocando en una cápsula Petri una cantidad de la soya desactivada o harina de soya que cubra el fondo de la cápsula y se agrega la solución rojo de fenol hasta que quede humedecido sin llegar a estar sobre cargado de líquido y se verifican los puntos rojos formados en el fondo a los cinco minutos y, luego de 25 minutos, mientras menos puntos aparezcan mejor esta la soya.

\section{f) Obtener información sobre los líquidos usados en la formulación \\ Los líquidos utilizados para la} fórmulas de los alimentos son metionina o alimex (Pinto et al. 2003) que es un aminoácido necesario para el organismo de las ponedoras, así mismo se utiliza colina que es un complejo vitamínico B12 y la grasa que va dependiendo de cuál es la que se encuentra en planta se propone la cantidad a utilizar, las grasa que entran en la realización del alimento son oleína, grasa amarilla, aceite de palma rojo, aceite crudo de soya, etc. Se elabora la ficha técnica o toxicología de cada uno de los fluidos necesarios para la alimentación.

\section{g) Establecer contacto directo con las mezcladoras y ubicar los dosificadores de líquidos para la verificación}

En la planta de alimentos existen dos mezcladoras (M-1 y M-2). Éstas son reactores batch (Darnoko y Cheryan, 2000) y funcionan cargando toda la materia prima, dividida en los granos como son maíz y soya. Todos los polvos y granos más pequeños son cargados en la tolva, luego agregan los líquidos y mezclan durante seis minutos hasta descargar y transportar a los silos, a través de tornillos sin fin. Se considera que la planta en su mayor parte es de proceso manual, por ello los líquidos usados para los alimentos balanceados son dosificados a través de un temporizador y en un determinado tiempo se desactiva agregando lo necesario a la mezcladora. Los líquidos utilizados la colina y la grasa son procesos manuales y la metionina o alimex es un proceso automatizado y, en tal sentido, el error es mucho menor. Los dosificadores se encuentran ubicados de la siguiente 
manera en la parte superior de las mezcladoras es agregada la colina, en la parte frontal se encuentran los dosificadores del alimex y en la lateral se encuentra el tanque de grasas, todas los dosificadores poseen con sus respectivas conexiones.

\section{h) Riesgos; consecuencias de una incorrecta dosificación de líquidos}

Una incorrecta dosificación consiste en una mala administración de los fluidos agregando mayor o menor cantidad de lo estipulado en las fórmulas nutricionales. La empresa Alimentos La Caridad, C.A. planta Valencia está encaminada a ser una empresa de calidad de productos alimenticios balanceados para aves como lo aporta su misión y uno de sus valores es ser perfecto o llegar a la perfección lo más cercano posibles, sin embargo mayor calidad en producto no significa mayor cantidad de materia prima, pero si hablamos de calidad, el agregar menor cantidades de materias primas es bajar calidad de la producción.

\section{i) Fórmulas nutricionales: cantidad de líquidos a dosar}

Se realizan las formulaciones nutricionales para que las gallinas tengan huevos de aproximadamente $65 \mathrm{~g}$, la consistencia cáscara que no sean frágil y tenga un color familiar, entre otros aspectos. Las fórmulas varían constantemente. Este cambio va sujeto a la materia prima existente (Dos Santos et al., 2003). En este sentido, las materias primas son recibidas y evaluadas, realizando análisis químicos como porcentaje de grasa, proteínas, calcio fósforo, entre otros, característicos de cada materia prima.
Estos análisis son reportado a los médicos nutricionistas, para que sean capases de desarrollar la fórmula de las cantidades adecuadas para un alimento de alta calidad. La cantidad de líquidos agregados al elaborar alimentos balanceados para aves va en conjunto con la cantidad de nutrientes de las materias primas obtenidas, es decir, son utilizados como complementos de una dieta balanceada y de eficaz actuación en la postura de las pollitas.

\section{j) Pruebas de dosificación de líquidos}

Las pruebas se realizan de manera triplicada para validar los valores utilizando tres tobos distintos, con una misma capacidad de $18 \mathrm{l}$. Se comienza por una mezcladora pesando los tres tobos y colocándolos en orden para así comenzar el proceso de dosificación. Este proceso requiere de cuidado, ya que se manipulan llaves o válvulas que pueden obstruir el paso del líquido a las mezcladoras y si no se colocan como deberían, entonces genera un error humano en el proceso de elaboración de alimentos. El estándar de procesos y técnicas realizado para la optimización de este proceso se encuentra descrito en el siguiente capítulo, el mismo sirve de ayuda a un personal no capacitado para realizar dichas pruebas, ya que es un proceso fácil, sencillo, preciso y muy bien especificado.

\section{k) Verificar variables peso del líquido) y el tiempo al dosar las mezcladoras}

Las variables cuantitativas medibles en las pruebas de dosificación son el peso de cada uno de los líquidos y el tiempo que tarda en dosar las mezcladoras se realiza 
para determinar la cantidad de líquido que es mezclado en el alimento balanceado. Los cálculos del peso neto se realiza con el tobo vacío, posteriormente se pesa lleno con la muestra obtenida para realizar una diferencia de los valores ya obtenidos. El valor de diferencia tiende a compararse con el esperado o formulado, de allí se obtiene la desviación que existe en el proceso y derivado para el proceso de verificación de las mezcladoras en planta con ayuda del personal del departamento de mecánica. Este proceso se realiza para todas las muestras, obteniendo tres de metionina y colina, líquidos utilizados en la fórmula nutricional del alimento balanceado para aves. Estos son los fluidos utilizados con menos cantidad dentro del alimento, de la grasa se realizan sólo un duplicado.

\section{l) Reporte la desviación al dosar de cada mezcladora}

Los valores obtenidos de las pruebas de dosificación y la determinación de valor neto y de desviación, así como también el tiempo de cada tomado de muestra son reportados mediante una hoja al jefe del laboratorio de aseguramiento de la caridad. Si es afirmativa la anomalía en el proceso de dosificación los encargados buscan una solución pertinente para corregirlo.

\section{m) Inspeccionar y notificar al supervisor de laboratorio sobre cualquier alteración en las muestras}

La inspección en la dosificación de líquidos, productos terminados y materias primas deben ser constantes debido a que de allí se ramifican la razón de ser del laboratorio de aseguramiento de la calidad, cualquier anormalidad que sea observada en los resultados de los análisis deben ser notificados a los entes superiores para conciliar un solución rápida y efectiva.

\section{RESULTADOS}

La Figura 1 muestra las cuatro fases llevadas a cabo para lograr el propósito del trabajo. Estas fases se describen a continuación:

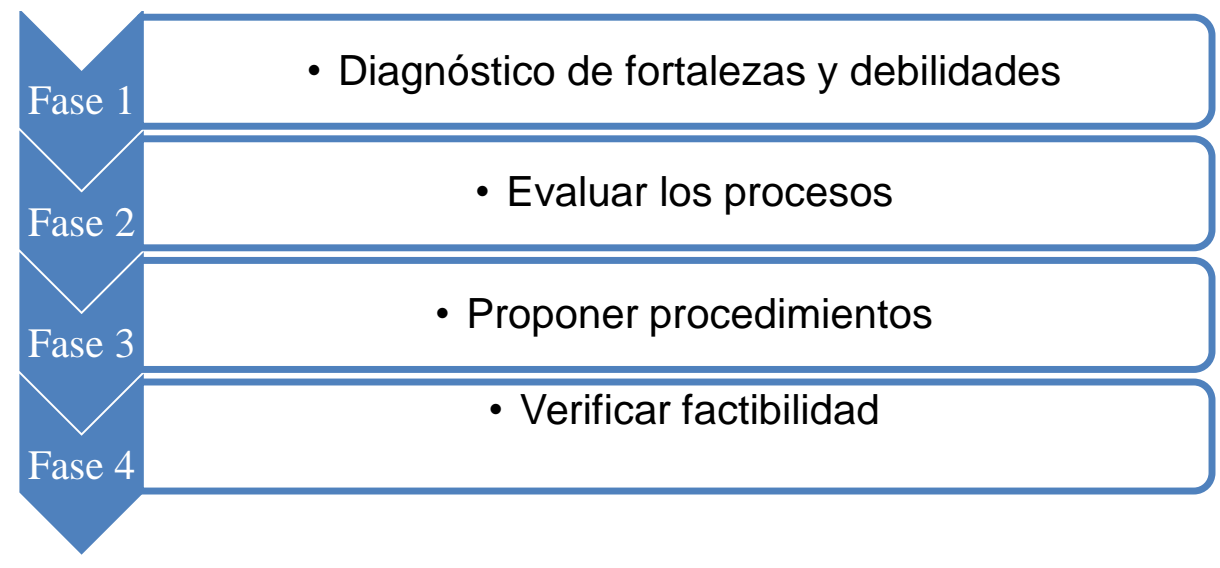

Figura 1. Fases del trabajo 


\section{Fase 1: Diagnóstico de fortalezas y} debilidades

Para el diagnóstico se localiza la información referente a las especificaciones de cómo realizar el procedimiento, cuáles son cada uno de los líquidos utilizados, sus características y propiedades, toxicología de los fluidos, consecuencias de una mala dosificación entre otras investigaciones realizadas donde se percibió la importancia del cumplimiento de una buena adición de los líquidos para certificar la calidad de un $100 \%$ del alimento formulado en la empresa, ya que dentro de la misión está ubicarse en una de las mejores empresas productoras de alimentos balanceados para aves ponedoras del mundo. Una mala dosificación puede repercutir en el nivel de posturas de estas aves, este es un error catastrófico en una granja de ponedoras y esto se debe a que los líquidos agregados en la formulación del alimento son complementos multivitamínicos necesarios para el organismo de estos animales y para poder producir un huevo de excelente calidad, dentro de los estándares de producción.

Una de las propiedades importantes para cumplir con los estándares de calidad de un huevo es la alimentación y las vitaminas consumidas $\mathrm{y}$ los líquidos dosados. La colina es un complejo vitamínico dentro del grupo $B$, que son capaces de extraer las proteínas a un máximo porcentaje de los alimentos generando un mayor aprovechamiento. La metionina es un aminoácido esencial que ayuda con el procesamiento de algunas toxinas no deseadas y, por último, están las grasas que son necesarias para el organismo, tanto animal como de un ser humano.

\section{Fase 2: Evaluar el proceso}

En esta etapa se evalúan los procedimientos haciendo contacto directo con cada una de las mezcladoras, indagando su funcionamiento, proceso de mezcla, dosificadores de cada uno de los líquidos entre otros. Se establece conexión con los operadores y obreros de la planta que son parte fundamental para culminar esta tarea, ya que, con tantos años de arduo trabajos dentro de la industria, conocen de cerca el proceso de dosificación, cómo funcionan las mezcladoras y que sucede dentro de planta al no dosar las cantidades necesarias. Los procedimientos para realizar la dosificación son engorrosos y levemente peligrosos, esto se debe a que las labores de dosificación o pruebas de dosificación se realizan en alturas.

Se visualiza el dosificador de grasa que posee un estándar de desviación más elevado, este puede darse por el error humano, pues el operador agrega la misma presionando el botón de dosificación y con una romana interna que posee el tanque de almacenamiento de grasas ubicado en la parte lateral izquierda de cada una de las mezcladoras, y en el panel de control se va verificando cuanta cantidad de peso pierde el tanque de almacenamiento $\mathrm{y}$ puede agregarse mayor o menor cantidad, otra desviación existente en este sistema es la cantidad que se mantiene adherida a las paredes de las tuberías de dosificación se estipula alrededor de $1.000 \mathrm{~kg}$ de perdida en las paredes internas de las tuberías.

Para la verificación de la metionina se poseen ocho llaves $o$ válvulas para manipular y obtener las pruebas de dosificación, en este líquido su desviación tiende a ser muy baja, debido a que la 
planta de metionina automatizada, y su valor esperado es muy cercano al obtenido en las pruebas o verificación de la misma. Para cumplir con esta etapa se conoce, de manera muy profunda, las mezcladoras, así mismo se observa el recorrido de las tuberías para la agregación de los líquidos, también se inspeccionan los dosificadores físicamente evaluando su aspecto, si existe alguna fuga, para dar fin a esta fase cumplida se estableció que por razones de seguridad las mezcladoras deben estar apagadas totalmente para evitar cualquier evento no deseado.

\section{Fase 3: Proponer procedimientos}

Velásquez et al. (2017) realiza una propuesta de los procedimientos y las técnicas que se deben ejecutar para que la dosificación sea fluida y fácil para el personal encargado de tomar muestras, en ella se expresa de manera explícita los pasos a seguir para realizar la dosificación, los equipos de seguridad necesarias, los cálculos para la obtención del valor neto, entre otros. Estas normas fueron estipuladas con más exactitud para que cualquier persona bien sea nuevo ingreso o ajena al laboratorio pueda entender claramente el proceso de dosificación. En el plan de procedimientos y técnicas se establecen claramente dónde se encuentran los dosificadores con ayuda de referencia fotográficas y se observan detalladamente las válvulas o llaves a manipular en cada uno de los sistemas dosificadores de los líquidos antes mencionados.

La verificación se realiza triplicados para obtener valores más concretos comparando los tres tomando en cuenta el tiempo de dosificación en minutos. Esta tercera fase lo que busco fue aportar herramientas más útiles al analista o auxiliar del laboratorio a la hora de realizar la verificación correspondiente que es muy necesaria para cumplir con un alto porcentaje de calidad en la producción de alimentos. A continuación en el anexo 11 de se observa el plan de dosificación estipulado para los trabajadores de la empresa específicamente a los empleados del laboratorio de aseguramiento de la calidad.

\section{Fase 4: Factibilidad}

Se hacen las pruebas de factibilidad de la propuesta de los procedimientos $\mathrm{y}$ técnicas para mejorar el proceso de dosificación de los líquidos, aportando normas para la ejecución de la misma, la dosificación se realizaba entre cada 15-21 días anteriormente en esta etapa se evaluó la factibilidad de realizar el muestreo cada semana para controlar el proceso más consecutivamente y evitar que el sistema sufra un descontrol en su configuración en cada uno de los equipos. Se verificó la factibilidad de manipular solo cuatro de las válvulas o llaves en la planta automatizada de metionina, de las cuales dos son provenientes de la tubería madre y cierra el paso a las ramificaciones y las otras dos se abren para dar paso al fluido y obtener las muestras.

\section{CONCLUSIONES}

En los alimentos balanceados para aves los nutrientes juegan parte fundamental de una alimentación saludable. En las gallinas ponedoras, el alimento proporciona la cualidad a las pollitas de poner huevos sin necesidad de ser apareada por un gallo. Por ello es necesario que la producción de estos alimentos sea de la más alta calidad para 
que así puedan proporcionales vitaminas y minerales. En la elaboración de los sustentos alimenticios se encuentra inmerso el proceso de agregación de los líquidos necesarios para complementar los compuestos vitamínicos del mismo.

El proceso de dosificación consiste en estandarizar la desviación que existe al agregar los compuestos líquidos y que el error sea menor, los líquidos utilizados son la colina, metionina y las grasas cada uno de ellos cumple un papel importante para la absorción y aprovechamiento de los nutrientes promovido por los cereales, las vitaminas o pre-mezclas y los polvos agregados. Por ello es relevante mantener una constante verificación para que así el proceso produzca un error mínimo y los alimentos producidos en la planta sean de mayor provecho para estos animales. La elaboración de una estandarización de procedimientos y técnicas de la dosificación de líquidos de las mezcladoras M-1 y M-2, el mismo sirve como un instrumento que permita la descripción del proceso de dosificación de líquidos realizado en la empresa Alimentos La Caridad, C.A. El mismo está estructurado bajo los lineamientos de la empresa. De esta manera se puede afirmar que la propuesta realizada, sobre los procedimientos y las técnicas para la realización de este proceso, fue verificada de forma técnica su factibilidad, arrojando resultados satisfactorios en la adición de líquidos en las mezcladoras.

De esta manera se puede dar fe que se logró cumplir con los objetivos planteados para culminar el informe como eran el diagnóstico de las fortalezas y debilidades de la dosificación de líquidos, la evaluación del proceso de dosificación realizado actualmente, la propuesta de la estandarización de procedimientos y técnicas para cumplir con lo cometido y la verificación técnica de la factibilidad del procedimiento con las técnicas $\mathrm{y}$ procedimientos propuesto, todo lo realizado obteniendo una final satisfactorio cumpliendo con las necesidades del laboratorio para que el proceso de verificación de la adición de líquidos sea más precisa y menos tediosa.

\section{REFERENCIAS}

Acosta, A., y Cárdenas, M. (2006). Enzimas en la alimentación de las aves. Fitasas. Revista Cubana de Ciencia Agrícola, 40(4), 377-387

Antelo, B. (2008). Efecto del tiempo y procedimiento de mezclado del alimento balanceado para gallinas ponedoras sobre la producción y calidad del huevo de mesa. Zamorano, Tegucigalpa: Escuela Agrícola Panamericana

Boué, L. M. E., Croublet, A. B. B., Pérez, M. D., y del Valle Arnaud, E. (2010). Evaluación de Agar Urea de Christensen modificado. Revista Información Científica, 68(4)

Castello, J. A. (1977).Nutrición de las aves (No. 84-85110-17-X. 03-A2 LU. PPNTR. 7.). Ediciones Sertebi

Castillo Rodríguez, D. C. (2017). Pollos de cuello desnudo, alimentados con harina de fréjol (Phaseolus vulgaris) germinado Quevedo: UTEQ

COVENIN (1980) .COVENIN 1567-80. Alimentos para aves. Método de Muestreo. Caracas: Normas venezolanas

Darnoko, D., y Cheryan, M. (2000). Kinetics of palm oil transesterification in a batch reactor. Journal of the American Oil Chemists' Society, 77(12), 12631267

Dos Santos, A. L., Callejo, A., Nicodemus, N., Villamide, M. J., Gutiérres, A., y Buxade, C. (2013). Exigencia nutricional de treonina digestible para gallina 
ponedoras durante el período de 55 a 61 semanas de edad. 1. Nivel de producción. En Memorias Congreso Científico de Avicultura

Farfán López, C. J., y Gordón, G. (2013). Evaluación nutricional de una mezcla de harina de maíz con harina de víscera y harina de sangre y plumas utilizada en la alimentación de aves. Zootecnia tropical, 31(2), 111-117

Fonseca, D. M. S., y Fonseca, J. A. (2011). Producción sostenible de pollo de engorde y gallina ponedora campesina: revisión bibliográfica y propuesta de un modelo para pequeños productores. RIAA, 2(1), 29-43

Fuentes, O., y Heber, A. (2016). Efecto de la temperatura y velocidad de rotación del tornillo en el proceso de extrusión de maíz, quinua y avena para la elaboración de harina pregelatinizada. Zamorano: Escuela Agrícola Panamericana, Tegucigalpa

Hidalgo, K., y Rodríguez, B. (2015). La alimentación de las aves, cincuenta años de investigaciones en el Instituto de Ciencia Animal. Revista Cubana de Ciencia Agrícola,49(2), 197-204

Marín-Gómez, S. Y., y Benavides-Montaño, J. A. (2007). Parásitos en aves domésticas (Gallus domesticus) en el Noroccidente de Colombia. Veterinaria y Zootecnia,1(2), 43-51

Martín, J. C. (2005). Alimentación de la pollita y la ponedora comercial: programas prácticos. Toledo: Casirrubios del Norte

Martínez, M., Moschini, R., Barreto, D., Bodega, J., Comerio, R., Forjan, H., y Valentinuz, O. (2010). Factores ambientales que afectan el contenido de fumonisina en granos de maíz. Tropical Plant Pathology,35(5), 277284
Meleán-Romero, R., Bonomie-Sánchez, M. E., y Rodríguez-Medina, G. (2008). Procesos productivos de la industria avícola zuliana: Fases de alimento, engorde y beneficio. Revista de la Facultad de Agronomía, 25(1), 160-184

Mendoza León, J. A. (2016). Evaluación de cuatro dietas alimentarias, en fase de crecimiento de pollos camperos traspatio; en el recinto Casa Azul del cantón Vinces. Universidad de Guayaquil; Facultad de Ciencias para el Desarrollo

Pinto, R., Donzele, J. L., Ferreira, A. S., Albino, L. F. T., Soares, R. D. T. R. N., de Almeida, M., y Silva, T. A. P. (2003). Exigência de metionina mais cistina para codornas japonesas em postura. Revista Brasileira de Zootecnia, 32(5), 1166-1173

Tovar, C. D. G., y Colonia, B. S. O. (2013). Producción y procesamiento del maíz en Colombia. Revista Guillermo de Ockham, 11(1), 97-110

Valdivié, M., Rodríguez, B., y Bernal, H. (2008). Alimentación de cerdos, aves y conejos con plátano (Musa paradisiaca L.).Rev. $A C P A, 1(2), 48-50$

Vallortigara, G., Zanforlin, M., y Pasti, G. (1990). Geometric modules in animals' spatial representations: a test with chicks (Gallus gallus domesticus). Journal of Comparative Psychology, 104(3), 248

Velasquez, M., Gómez, Y y Duarte, Y (2017). Estandarización de los procedimientos y técnicas en la dosificación de líquidos de las mezcladoras $\mathrm{m} 1$ y $\mathrm{m} 2$, para la formulación de alimentos balaceados para aves, en la empresa Alimentos La Caridad C.A, ubicada en Valencia, estado Carabobo. UNEFA 